\title{
EL PROBLEMA DEL CAMBIO \\ EN LAS CONCEPCIONES DE ESTUDIANTES DE FORMACIÓN AVANZADA
}

\author{
GALLEGO BADILLO, RÓMULO y PÉREZ MIRANDA, ROYMAN \\ Universidad Pedagógica Nacional. Bogotá, D.C. Colombia
}

\begin{abstract}
Resumen. Este artículo presenta las conclusiones obtenidas con un grupo de diecisiete profesores estudiantes de química sobre las evoluciones de las concepciones epistemológicas y didácticas en el primer año de formación en el programa de Maestría en docencia de la Química.
\end{abstract}

Palabras clave. Concepciones de profesores, evolución de conceptos, enseñabilidad, educabilidad, educación en ciencias.

Summary. This article presents the conclusion obtained with a group of seventeen Chemistry teachers on the evolution of epistemological, educational and didactic conceptions during the first year of a programme leading to a M.S. (Teaching of Chemistry).

Keywords. Teacher's conception, evolution of concepts, ensenability, educability, science education.

\section{INTRODUCCIÓN}

La presente investigación se realizó con estudiantes del programa de maestría en docencia de la química, del Departamento de Química de la Universidad Pedagógica Nacional, todos ellos con formación inicial como profesores de ciencias, que además ejercían como tales en instituciones educativas de Bogotá.

La preocupación por las concepciones de los profesores en ejercicio, tanto como por la transformación de las mismas a través de procesos pedagógicos y didácticos diseñados con tal objetivo, se inicia entre quienes son los responsables de la presente investigación hace ya un lustro (Gallego Badillo, Pérez Miranda y Urrea, 1995). En este sentido se inscribe en una tradición de trabajos tales como los referenciados por Porlán, Rivero y Martín del Pozo (1997) y Lederman (1992). Este ámbito de investigación es también conocido como el «del pensamiento del profesor»(Gallego Arrufat, 1991), el cual está adquiriendo cada vez mayor importancia entre los investigadores en la enseñanza de las ciencias (Mellado, 1996). Se han centrado, muchos de esos trabajos, en las creencias de los profesores y en sus concepciones epis- temológicas. En este orden de ideas se ha puesto de manifiesto que el profesorado de estas disciplinas posee una discutible comprensión de la naturaleza de las ciencias y de las características del trabajo científico. Tal apreciación ha llevado a sugerir que el mejoramiento del proceso de enseñanza y de aprendizaje sólo es factible si se modifican las ideas epistemológicas del propio profesorado (Carrascosa, Fernández, Gil y Orozco, 1993).

En este orden de ideas, parece haber claridad con respecto a que el pensamiento de los profesores debe ser objeto de trabajo fundamental y prioritario en el interior de un proceso de formación permanente, por lo que podría afirmarse que las ideas que ellos construyen a lo largo de sus historias profesionales se expresan y se caracterizan por un cuerpo de conceptos, valores y creencias organizados en teorías explícitas o implícitas, que se concretan en unas estrategias y unos métodos de actuación (Pérez Gómez, 1987). En general, es sostenible que los profesores tienen representaciones complejas y a veces ambivalentes acerca de la enseñanza de las ciencias experimentales (Boyer y Thiberghein, 1989). Estos últimos 
investigadores resaltan, en esos profesores, la existencia de un modelo educativo centrado en la transmisión de conocimientos, modelo en el que las disciplinas científicas no son constructos de la realidad elaborados epistemológicamente $\mathrm{y}$, por tanto, tampoco son asumidos como sistemas sociales en cuya construcción inciden interferencias múltiples.

En consecuencia, se partió de la convicción de que el grupo de estudiantes del programa de maestría señalado había construido unas concepciones epistemológicas, pedagógicas y didácticas en su formación inicial -en la que habría predominado una aproximación empiropositivista y el paradigma de la transmisión-repetición-, formación esta en la que bastaba saber la ciencia correspondiente para enseñarla. Además, este paradigma se había afianzado con la práctica profesional, como profesores de ciencias, ejercida en las diferentes instituciones escolares.

Conocidos los programas de formación inicial mediante los cuales estos estudiantes de maestría obtuvieron su título de licenciado, se concluyó que la mayoría de ellos no habían tenido la oportunidad de estudiar y discutir las nuevas versiones epistemológicas sobre la naturaleza de las ciencias experimentales y que las ideas que habían elaborado al respecto procedían de los textos didácticos acerca de la química o de la biología. Estos textos les habrían permitido acceder a una versión de las estructuras de dichas ciencias, al mismo tiempo que a una organización didáctica de ellas. En cuanto a la formación en esta última y en la pedagogía de dichas disciplinas éstas provenían de cursos adicionales, por lo general de carácter instrumental, en los que no se examinaban las formas como se les habían enseñado las disciplinas químicas y biológicas.

Para identificar y examinar los conceptos epistemológicos, pedagógicos y didácticos de los estudiantes, vía las transformaciones necesarias, el marco teórico de partida en cuanto a lo epistemológico fue el de las aproximaciones deductivistas constructivistas basadas en los aportes de Popper (1962), Kuhn (1972) y Lakatos (1983), sobre todo los dos últimos por sus basamentos históricos. En relación con lo pedagógico, se fundamentó en las ideas acerca de la educación en ciencias y en la conceptualización en torno al aprendizaje como cambio conceptual, metodológico, actitudinal y axiológico (Gallego Badillo y Pérez Miranda, 1994). En lo referente a la didáctica, se partió del examen de los distintos modelos de enseñanza (Gil, 1983) y se adoptó aquél basado en unas estrategias que perseguían el reconocimiento crítico de lo que los estudiantes sabían al respecto, con miras a una reconstrucción de sus ideas previas. En este contex to primó que enseñar es formular y poner en práctica un ámbito didáctico para propiciar experiencias de aprendizaje (Gallego Badillo y Pérez Miranda, 1997).

Tan criticable como se quiera, la investigación fue direccionada por los supuestos de que, en el caso de las ciencias, la pedagogía se ocupaba de los problemas de la educación, de lo educativo y de la educabilidad que ellas propician (Gallego Badillo y Pérez Miranda, 1999). Y su didáctica, por los también problemas acerca de la enseñabilidad y la enseñanza, condicionadas por las concepciones histórico epistemológicas elaboradas por los profesores como consecuencia de su formación inicial.

De acuerdo con lo anterior, el problema abocado fue el de identificar las concepciones epistemológicas, pedagógicas y didácticas de estos estudiantes, analizar la coherencia entre ellas y diseñar unas estrategias didácticas con miras a lograr transformaciones que se aproximen a las posiciones constructivistas.

\section{METODOLOGÍA}

Se describen a continuación el grupo con el cual se adelantó la investigación, los instrumentos que se diseñaron y aplicaron para tal efecto y las estrategias pedagógicas y didácticas empleadas.

\section{Grupo con el cual se adelantó la investigación}

Este grupo estuvo constituido por los estudiantes admitidos en el Programa de Maestría en Docencia de la Química, del Departamento de Química de la Universidad Pedagógica Nacional, en el año de 1996. El número total fue de 17. Para una mejor ilustración de las características del grupo ver tabla I (Anexo I).

\section{Selección y diseño de los instrumentos}

Para la identificación de las ideas de los estudiantes, se emplearon diferentes técnicas (Barsese, Lumbaca y Pentamalli, 1996). La construcción y la validación de tales pruebas se fundamentaron en la aceptación de que la información que suministraran fuera relevante para la formulación y praxis de las estrategias didácticas; información que se presumió no trivial (Lang de Silveira y Moreira, 1996). La convicción fue la de que el empleo de una batería de pruebas diferentes sobre lo mismo, cruzando los resultados obtenidos con cada una de ellas, suministraba mayor y más completa información para la emisión de un juicio válido, en torno a la obtención de los objetivos de la presente investigación. Se recogió lo recomendado por Barquín (1995), en cuanto al problema del diseño de instrumentos para la identificación de las concepciones de los estudiantes, a la vez que se acudió a las pruebas tipo Likert, siguiendo las modificaciones introducidas por Porlán (1989) tanto para identificar y analizar tendencias alrededor de las concepciones epistemológicas, pedagógicas y didácticas del grupo como para el análisis crítico de tales tendencias.

Las pruebas tipo Likert fueron de dos clases: $a$ ) una con miras a identificar y a analizar las concepciones ya anotadas en relación con las ciencias experimentales en general (Anexo II); y $b$ ) otra referida a la química en particular (Anexo III).

En consecuencia con los presupuestos metodológicos enunciados, además de las pruebas tipo Likert, se proce- 
dió al diseño y aplicación de las siguientes pruebas: semántica (Anexo IV), composición, ensayos, elaboración de mapas conceptuales y resolución de un cuestionario (Anexo V). Para la elaboración de la prueba semántica, se seleccionaron unas categorías centrales, relacionadas con la naturaleza de las ciencias en general, y de la química en particular, sus enseñanzas y sus aprendizajes, lista que se suministró a cada uno de los estudiantes, solicitándoles que expresaran por escrito lo que le significaba cada categoría.

Las categorías seleccionadas constituyeron la base para la elaboración de mapas conceptuales (Novak y Gowin, 1988), categorías que a su vez fueron fundamento para que ellos, los estudiantes, redactaran composiciones que debían titular. Este título traduciría objetivos y, por tanto, intereses y, en cierta manera, actitudes. Las pruebas descritas fueron aplicadas al iniciar y al concluir el proceso durante ese primer año lectivo, con excepción del cuestionario que resolvieron al final, en el programa de Maestría en docencia de la Química.

En lo referente al cuestionario, se siguió lo recomendado por Briones (1988), por lo que fue de preguntas abiertas. Las preguntas buscaron auscultar la incidencia del trabajo pedagógico y didáctico desarrollado a lo largo del año en referencia al desempeño docente de cada uno de los miembros del grupo. Los resultados de tales pruebas fueron analizados desde el marco conceptual y metodológico que ha sido explicitado.

Para la elaboración, tanto de las pruebas tipo Likert como para la formulación de las categorías, se especificaron tres campos: el de lo epistemológico, el de lo pedagógico y el de la didáctica; discriminándose en cada uno de ellos dos tendencias, la empriropositivista y la constructivista.

\section{Estrategias pedagógicas y didácticas}

Ésta se centraron en la labor de enseñar a leer, a escribir y a sustentar verbalmente las interpretaciones de los textos recomendados para la comprensión tanto de las posiciones epistemológicas actuales como de las relativas a las ideas acerca de la enseñanza y el aprendizaje de las ciencias experimentales que predominaban en su momento. Implicó, igualmente, concitar a los estudiantes de la maestría a confrontar sus concepciones previas con las propias de los autores estudiados.

Estas estrategias pedagógicas y didácticas giraron alrededor, fundamentalmente, de dos seminarios programados en el plan de estudio: el primero, Epistemología e Historia de la Química y, el segundo, Teorías del Aprendizaje y Enseñanza de las Ciencias. En el primero fueron objeto de estudio y discusión, Bacon (1979), Popper (1962), Kuhn (1972) y Lakatos (1983). Cada uno de los estudiantes debía presentar, antes de abordar la discusión, de cada autor en el grupo, un ensayo sobre la obra objeto de análisis. Para la finalización de este seminario, se les solicitó la elaboración de un trabajo en el que cada estudiante debía proponer unas respuestas admisibles a los siguientes interrogantes: ¿Es la química una ciencia experimental? ¿Por qué? ¿Son factibles una pedagogía y una didáctica de la química así concebida?

Para centrar las reflexiones epistemológicas se estudió el desarrollo y la evolución histórica de teorías químicas. Se seleccionaron las siguientes temáticas, para ser abordadas, cada una de ellas, por grupos según los intereses de esos estudiantes: $a$ ) las teorías del flogisto y de la oxidación; $b$ ) la teoría atómica; $c$ ) la teoría estructuralista de Berzelius; $d$ ) las teorías de enlace; y $e$ ) las teorías ácido-base. Como preámbulo a esa revisión histórica, se discutió en torno a la diferencia entre una historiografía de descubrimientos y un estudio basado en la existencia de teorías rivales, de paradigmas en competencias o de programas de investigación competitivos. Al respecto se revisó el texto de Thuiller (1975). El interrogante crucial fue aquél que preguntó por la existencia de teorías propiamente químicas.

En relación con el seminario Teorías de Aprendizaje y Enseñanza de las Ciencias, éste giró alrededor de un análisis crítico de cinco posiciones, respecto al problema del aprendizaje: a) la concepción transmisionistarepeticionista, centrada en los postulados del conductismo operante y la tecnología educativa (Skinner, 1986); b) las concepciones derivadas de la epistemología genética de Piaget y las variaciones sobre el desarrollo del pensamiento (Piaget, 1985; Inhelder y Piaget, 1972); c) las concepciones surgidas del aprendizaje social, enmarcadas en las afirmaciones de Vygotski (1989), teniendo como eje problemático las zonas de desarrollo real y de desarrollo próximo; $d$ ) las concepciones de aprendizaje significativo de Ausubel, Novak y Hanesian (1983) con las variaciones formuladas por Novak y Gowin $(1988) ; e$ ) por último, la concepción de aprendizaje sostenido como la del cambio, con toda la evolución histórica que ha venido reestructurándola (Posner, Strike, Hewson y Gertzog, 1982; Gil y Carrascosa, 1985 y Gené, 1991), hasta el posicionamiento y postulados del programa de investigación que dirigen los autores del presente artículo (1994), sobre el aprendizaje como cambio conceptual, metodológico, actitudinal y axiológico, en la perspectiva de un aprendizaje total (Gallego Badillo, Pérez Miranda y Torres de Gallego, 1997). Este último está basado en un triple reconocimiento, que ha de hacer quien se halla involucrado en el proceso: ¿qué es lo que aprende? ¿cómo lo aprende? y que es él quien lo aprende, y ha de responder por ello, en los significados, formas de significar y de actuar que está construyendo.

Al iniciar este seminario se formularon tres interrogantes que direccionaron su desarrollo: ¿Es la química aprendible? ¿Es enseñable? ¿Por qué? Sobre estos interrogantes cada uno de los participantes debía elaborar un trabajo final en el cual diera unas respuestas admisibles y un posicionamiento teórico al respecto.

En cuanto a la enseñanza de las ciencias y su relación con las teorías de aprendizaje, se solicitó a cada uno de los comprometidos, una vez terminadas las cinco temáticas propuestas, que redactara un ensayo en el que intentara mostrar las posibilidades de desarrollar críticamente una 
didáctica de las ciencias experimentales, en general, y de la química, en particular, desde los postulados trabajados, expresable en la producción concreta de un diseño de estrategias para el trabajo en el aula de clase con los estudiantes, con los cuales ejercía, en aquella época, su labor docente. La intencionalidad final era la de que mudaran hacia las concepciones constructivistas sobre la enseñanza y el aprendizaje.

\section{Análisis de los resultados obtenidos}

Se procedió como se expone a continuación. Con las pruebas tipo Likert sobre la ciencia, en general, y en relación con las tendencias sobre la enseñanza, el aprendizaje y las concepciones epistemológicas de los estudiantes, se tomaron y promediaron las puntuaciones de las siguientes afirmaciones:

- enseñanza empiropositivista: $1,6,9$,17, 28 y 30;

- enseñanza constructivista: 12, 21, 23 37, 42 y 48;

- aprendizaje empiropositivista: 3, 20, 25, 27, 36 y 43;

- aprendizaje constructivista: 5, 11, 15, 33, 40, 46 y 49;

- epistemología empiropositivista: 2, 8, 14, 19, 32, 35, 47 y 50

- epistemología constructivista: 4, 10, 16, 24, 29, 38 y 41.

En el caso de la prueba tipo Likert sobre química, se procedió de igual manera, tal como se enlista a continuación:
- epistemología empiropositivista: 4, 5, 14, 17, 18, 29, 45 y 46

- epistemología constructivista: 1, 6, 10, 13, 20, 30, 31, 35 y 41 ;

- enseñanza empiropositivista: 24, 34 y 39;

- enseñanza constructivista: 19 y 48;

- aprendizaje empiropositivista: 15, 25, 40 y 50;

- aprendizaje constructivista: 33 .

En el análisis de las pruebas semánticas y de composición, se tuvieron en cuenta, con respecto a las primeras, las posiciones epistemológica, pedagógica y didáctica que se desprendían de las definiciones iniciales y finales dadas a las categorías enlistadas. En cuanto a las últimas, el título que cada uno daba a sus composiciones, también iniciales y finales. En relación con los mapas conceptuales, se identificaron las relaciones simples y válidas entre conceptos, las relaciones cruzadas, las jerarquizaciones entre ellos y el uso de ejemplos. Cada mapa se puntuó de la siguiente manera (Novak, 1981): relaciones simples válidas entre conceptos: 1 punto por cada una; relaciones cruzadas: 5 puntos por cada una; jerarquizaciones entre conceptos: 5 puntos por cada una; uso de ejemplos: 1 punto por cada uno.

En las respuestas dadas al cuestionario final se examinaron también las tendencias que de ellas se deducían en relación con la permanencia o no de las concepciones epistemológicas, pedagógicas y didácticas, de tipo em-

Tabla II

Resultados promedios obtenidos en las pruebas tipo Likert.

\begin{tabular}{|l|c|c|c|c|}
\hline \multirow{2}{*}{ Concepciones } & \multicolumn{2}{|c|}{ Antes } & \multicolumn{2}{c|}{ Después } \\
\cline { 2 - 5 } & Empiropositivismo & Constructivismo & Empiropositivismo & Constructivismo \\
\hline Enseñanza & 3,9 & 2,2 & 4,2 & 2,3 \\
\hline Aprendizaje & 4,0 & 2,3 & 4,2 & 1,8 \\
\hline Epistemología (general) & 3,2 & 2,7 & 3,9 & 2,4 \\
\hline Química (en particular) & 3,3 & 2,8 & 3,7 & 2,5 \\
\hline
\end{tabular}

Tabla III

Frecuencias de tendencias en las pruebas semánticas del grupo.

\begin{tabular}{|l|c|c|c|c|}
\hline \multirow{2}{*}{ Categorías } & \multicolumn{2}{|c|}{ Antes } & \multicolumn{2}{c|}{ Después } \\
\cline { 2 - 5 } & Empiropositivismo & Constructivismo & Empiropositivismo & Constructivismo \\
\hline Ciencia & 15 & 2 & 8 & 9 \\
\hline Conocimiento & 14 & 3 & 9 & 7 \\
\hline Experimentación & 12 & 5 & 6 & 11 \\
\hline Teoría & 13 & 4 & 12 & 5 \\
\hline Enseñanza & 11 & 5 & 4 & 13 \\
\hline
\end{tabular}


Tabla IV

Resultados de los mapas conceptuales elaborados.

\begin{tabular}{|c|c|c|c|c|c|c|c|c|c|c|c|c|c|}
\hline Cat. & \multicolumn{2}{|c|}{ Rel. Val. } & \multicolumn{2}{|c|}{ Jerarquización } & \multicolumn{2}{|c|}{ Rel. cruzados } & \multicolumn{2}{|c|}{ Ejemplos } & \multicolumn{2}{|c|}{ Puntos } & \multicolumn{2}{|c|}{ Tipo de mapas } & \multicolumn{2}{c|}{ Avances } \\
\hline $\begin{array}{c}\text { Tiemp. } \\
\text { Est. }\end{array}$ & Antes & Después & Antes & Después & Antes & Después & Antes & Después & Antes & Después & Antes & Después & \\
\hline 01 & 4 & 6 & 3 & 2 & 0 & 1 & 0 & 0 & 19 & 21 & SI & HM & sí \\
\hline 02 & 0 & 11 & 4 & 5 & 0 & 2 & 0 & 0 & 20 & 41 & SI & HM & sí \\
\hline 03 & 1 & 6 & 4 & 4 & 0 & 0 & 0 & 0 & 21 & 26 & SI & SI & sí \\
\hline 04 & 0 & 7 & 4 & 3 & 1 & 3 & 0 & 0 & 25 & 32 & N & SI & no \\
\hline 05 & 0 & 11 & 5 & 4 & 0 & 1 & 0 & 0 & 25 & 36 & N & SI & sí \\
\hline 06 & 0 & 14 & 3 & 5 & 0 & 4 & 0 & 0 & 15 & 59 & N & SI & sí \\
\hline 07 & 0 & 17 & 5 & 5 & 0 & 0 & 0 & 0 & 25 & 42 & N & SI & sí \\
\hline 08 & 0 & 11 & 4 & 4 & 0 & 4 & 0 & 0 & 20 & 51 & N & SI & no \\
\hline 09 & 0 & 16 & 3 & 4 & 0 & 0 & 0 & 0 & 15 & 36 & N & HM & sí \\
\hline 10 & 0 & 13 & 3 & 5 & 0 & 0 & 0 & 4 & 15 & 60 & N & HM & sí \\
\hline 11 & 9 & 12 & 6 & 6 & 0 & 0 & 0 & 0 & 39 & 42 & SI & HM & sí \\
\hline 12 & 0 & 0 & 4 & 4 & 0 & 0 & 0 & 0 & 20 & 20 & N & N & no \\
\hline 13 & 0 & 7 & 3 & 4 & 0 & 2 & 0 & 0 & 15 & 37 & N & SI & no \\
\hline 14 & 4 & 6 & 2 & 4 & 0 & 1 & 0 & 0 & 14 & 31 & N & HM & sí \\
\hline 15 & 3 & 15 & 4 & 4 & 0 & 1 & 0 & 0 & 23 & 40 & N & HM & sí \\
\hline 16 & 19 & 15 & 5 & 3 & 1 & 0 & 0 & 0 & 49 & 30 & SI & SI & no \\
\hline 17 & 4 & 4 & 4 & 4 & 0 & 0 & 0 & 0 & 24 & 24 & SI & SI & no \\
\hline
\end{tabular}

SI: Se insinúa un mapa conceptual; HM: Expresa un modelo; N: No hay un modelo

piropositivista o constructivista, además de la incidencia que habían tenido las estrategias didácticas seguidas en relación con la perspectiva de cambio de cada uno de los estudiantes del grupo.

\section{RESULTADOS}

Siguiendo la lógica de los promedios establecida para los análisis, con respecto a las pruebas tipo Likert general y química en particular, la tabla II muestra los resultados globales obtenidos con los 17 estudiantes.

De las puntuaciones se pueden deducir las tendencias siguientes: en cuanto a la enseñanza antes se ve un desacuerdo en relación con los concepciones empiropositivista y un acuerdo con la constructivista. Los resultados finales señalan que no hubo un cambio significativo al respecto. En relación con la categoría de aprendizaje, los resultados iniciales y finales parecen seguir la misma tendencia que en el caso de la enseñanza, lo que muestra coherencia.
En lo referente a las concepciones epistemológicas, inicialmente hay tendencia a no saber qué decir, puesto que están alrededor de 3,0. Al final, esta situación se modifica en razón de que el grupo tiende a estar en desacuerdo con las concepciones empiropositivistas y, hasta cierto punto, de acuerdo con las constructivistas. Algo análogo hay que sostener en relación con las concepciones sobre la química como ciencia, con la tendencia final a estar en desacuerdo con las posiciones empiropositivistas y de acuerdo con las constructivistas. Las puntuaciones se ubican entre 2,0 y 3,0 aun cuando al final y en relación con las pruebas tipo Likert se puede afirmar que hubo cierta transformación en el grupo.

Los resultados parecen corroborar lo dicho, en el sentido de que no todo el grupo mudó de las posiciones empiropositivistas iniciales a las constructuivistas finales. Destáquese, por ejemplo, el caso de la categoría «teoría», en la que la mayoría del grupo se mantuvo en sus aproximaciones empiropositivistas, algo que introduce dudas en cuanto a la tendencia que muestran al respecto las pruebas tipo Likert, exceptuándose el caso de la categoría «enseñanza» en la que la mayoría mudó hacia posiciones constructivistas. De todos modos, la tabla III parece 
indicar la existencia de incoherencia cuando las puntuaciones de todas las categorías se comparan entre sí.

La lectura de los mapas conceptuales, después de puntuados, parece indicar, al final, el establecimiento, en primer lugar, de pocas jerarquizaciones y mínimas relaciones cruzadas; igualmente, y por lo general, la ausencia de ejemplos. Esto parecería indicar que, en relación con las categorías, los estudiantes no lograron estructurar una concepción epistemológica, pedagógica y didáctica, corroborándose así lo dicho en relación con las pruebas semánticas. No obstante, hubo cierto avance en lo mostrado en la construcción de un modelo explicativo para once de los diecisiete estudiantes (Tabla IV).

La tabla $\mathrm{V}$ informa que dos estudiantes no llevaron a cabo la titulación de su composición inicial, en tanto que uno de ellos no lo hizo en su composición final. En general, examinadas las composiciones y los títulos respectivos muestran un cambio significativo desde posiciones empiropositivistas a constructivistas, lo que contradice los resultados obtenidos con las pruebas semánticas, en las cuales el número de estudiantes que muestra una transformación hacia posiciones de tipo constructivista no sigue la misma proporción.

\section{De los ensayos presentados en el seminario sobre Epistemología e Historia de la Química}

Dígase que en la mayoría de los ensayos elaborados por los estudiantes, se limitaron a relatar lo que cada uno de los autores (Popper, Kuhn, Lakatos) sustentaban, por lo que no presentaron, los estudiantes, una posición crítica o una interpretación idiosincrática en la que pusieran por escrito sus ideas sobre la construcción históricoepistemológica de las ciencias experimentales. Algo análogo ocurrió en relación con el ensayo final en torno a la química como ciencia, la existencia de teorías propiamente químicas, la especificidad del experimento químico y la factibilidad de una pedagogía y una didáctica de la química, por lo que puede afirmarse que no lograron una transformación satisfactoria hacia posiciones constructivistas.

De los ensayos presentados en el seminario sobre Teorías de Aprendizaje y Enseñanza de las Ciencias

En este seminario que sigue al de Epistemología e Historia y de la Química y que, como consecuencia,

Tabla V

Resultados de las frecuencias en las tendencias del grupo en las composiciones.

\begin{tabular}{|l|c|c|}
\hline Momentos & & Constructivista \\
\hline Antes & 11 & 4 \\
\hline Después & 5 & 11 \\
\hline
\end{tabular}

constituye uno de los fundamentos del mismo, la evaluación de las propuestas escritas siguieron la misma tónica de los elaborados en el seminario anterior, limitándose, como quedó dicho, a relatar aquello de lo que los autores estudiados daban cuenta sin asumir posiciones críticas e interpretaciones idiosincráticas. La mayoría da por aceptado que la química es enseñable y aprendible sin más. Todo lo anterior permite puntualizar que el grupo, si bien en algunos aspectos mudó de su posición inicial con respecto al aprendizaje y la enseñanza de las ciencias en general, y de la química en particular, se consideró que no fue lo suficiente como para afirmar que se posicionaron epistemológica, pedagógica y didácticamente en una tendencia de carácter constructivista.

\section{Del cuestionario final}

El análisis de las respuestas dadas por el grupo permite afirmar que, en relación con la primera pregunta, en general, las respuestas no permiten inferir un cambio significativo en la mirada que cada uno de ellos tenía de su desempeño docente. En cuanto a la segunda, el estudio de las respuestas da pie para subrayar el posible logro de un cambio actitudinal frente a sus estudiantes y al trabajo en el aula. No señala al respecto que cambiaron desde las aproximaciones empiropositivistas a las constructivistas.

En lo referente a la tercera pregunta, las respuestas sugieren, en orden descendente, la estimación dada a las pruebas tipo Likert, las semánticas, las de composición y la de mapas conceptuales. En lo relacionado con la cuarta pregunta hay que subrayar la inscripción que lograron respecto de las futuras actuaciones didácticas, en la medida en que el grupo manifiesta la necesidad de conocer lo que los estudiantes ya saben en las temáticas de química y proceder de conformidad con esas ideas previas o alternativas (Furió, 1996).

Antes de cualquier conclusión se sostiene aquí que el estudio, identificación y transformación de las concepciones de los estudiantes constituye un problema que ha de ser mirado desde la perspectiva de una teoría de complejidad.

\section{CONCLUSIONES E IMPLICACIONES}

De acuerdo con los resultados obtenidos, y teniendo en cuenta el análisis realizado en todos los estudiantes, de las pruebas aplicadas, podría concluirse lo siguiente:

- El abordaje metodológico múltiple con las estrategias pedagógicas y didácticas descritas suministró la información necesaria para emitir un juicio sustentado sobre los aprendizajes, en términos de cambios conceptuales, metodológicos, actitudinales y axiológicos; cambios referidos a las concepciones epistemológicas, pedagógicas y didácticas, tanto de las ideas sobre la naturaleza de las ciencias experimentales, en general, como de la 
química, en particular, sus enseñanzas y sus aprendizajes con las que ingresaron los estudiantes al proceso.

- Este tipo de abordaje y de estrategias pedagógicas y didácticas suministró suficiente información para demostrar la persistencia de posiciones eclécticas en un porcentaje significativo de los sujetos, por lo que las estrategias aplicadas no surtieron el efecto perseguido. Hay, en consecuencia, concordancia con los resultados obtenidos por otros investigadores, tal como se referencian algunos de ellos en el marco conceptual y metodológico.

- Los resultados mostraron, en la mayoría de los casos, además, la existencia de disconcordancias entre las concepciones epistemológicas acerca de las ciencias experimentales en general, y de la química en particular, y las pedagógicas y didácticas, dado que hubo aproximaciones constructivistas en la primera categoría con tendencias a ideas sobre la enseñanza y el aprendizaje de tipo empiropositivista o, al contrario, constructivistas estas últimas y empiropositivistas las primeras. El proceso de cambio no ocurre de manera uniforme en cada una de tales categorías, como tampoco en cada uno de los estudiantes.
- Anótese al respecto que, en cuanto a los ensayos finales de los dos seminarios del plan de estudio del programa de Maestría en Docencia de la Química, éstos no fueron más que un conjunto de citas puntuales e inconexas de unos y otros autores. Todo ello podría tomarse como un cumplimiento acrítico de las tareas con la ausencia de interpretaciones idiosincráticas. De ahí que no aprendieron a leer y a escribir en epistemología, pedagogía y didáctica de las disciplinas estudiadas.

- Dentro de esta perspectiva, y dado que no se aproximaron a las lecturas recomendadas desde una mirada crítica y constructivista, parece ser que las actividades cognoscitivas impulsadas por estos seminarios no los movieron a elaborar una conceptualización desde la cual asumir que lo sostenido por los autores no son más que propuestas. De acuerdo con los resultados, persistió, en la mayoría de estos estudiantes, un eclecticismo ingenuo.

- Sería del caso reconocer que las teorías pedagógicas y didácticas de las ciencias experimentales, tomadas aquí como fundamento, acusan falencias que es preciso revisar y superar.

\section{REFERENCIAS BIBLIOGRÁFICAS}

AUSUBEL, D., NOVAK, J.D. y HANESIAN, H. (1983). Psicología educativa. Un punto de vista cognoscitivo. México: Trillas.

BACON, E. (1979). Novum Organom. México: Porrúa.

BARQUIN, J. (1995). La investigación sobre el profesorado. Estado de la cuestión en España. Revista de Educación, 306, pp. 7-65.

BARSESE, A., LUMBACA, P. y PENTIMALLI, R. (1996). Investigación sobre las concepciones de los estudiantes acerca de los estados de agregación y los cambios de estado. Enseñanza de las Ciencias, 14(1), pp. 14-24.

BOYER, R. y TIBERGHIEN, A. (1989). Las finalidades de la enseñanza de la física y la química vistas por profesores y alumnos franceses. Enseñanza de las Ciencias, 7(3), pp. 213-222.

BRIONES, G. (1988). Métodos y técnicas avanzados de investigación aplicados a la educación y a las ciencias sociales. Programa Interdisciplinario de Investigación en Educación. Módulo 3. Bogotá, ICFES-PIIG.

CARRASCOSA, J., FERNÁNDEZ, I., GIL, D. y OROZCO, A. (1993). Análisis de algunas visiones deformadas sobre la naturaleza de las ciencias y las características del trabajo científico. Enseñanza de las Ciencias, núm. extra, pp. 43-44.

FURIÓ, C. (1996). Las concepciones alternativas del alumno. Dos décadas de investigación. Resultados y tendencias. Alambique. Didáctica de las Ciencias Experimentales, 7, pp. 7-17

GALLEGO ARRUFAT, M.J. (1991). Investigación sobre pensamientos del profesor: aproximaciones al estudio de las teorías y creencias de los profesores. Revista Española de Pedagogía, 189, pp. 287-325.

GALLEGO BADILLO, R. y PÉREZ MIRANDA, R. (1999). Aprendibilidad, enseñabilidad y educabilidad en las ciencias experimentales. Educación y Pedagogía, XI, 25, pp. 87-117.

GALLEGO BADILLO, R, y PÉREZ MIRANDA, R, (1994). Representaciones y conceptos científicos. Un programa de investigación. Santafé de Bogotá: Universidad Pedagógica Nacional. Departamento de Química.

GALLEGO BADILLO, R. y PÉREZ MIRANDA, R. (1997). La enseñanza de las ciencias experimentales. Bogotá: Cooperativa Editorial Magisterio. 
GALLEGO BADILLO, R. PÉREZ MIRANDA, R. y TORRES DE GALLEGO, L.N. (1997). El aprendizaje total: Una aproximación teórica. Estudios en pedagogía y didáctica, 2(1), pp. 4-13.

GALLEGO BADILLO, R., PÉREZ MIRANDA, R. y URREA OSPINA, I.D. (1995). Concepciones epistemológicas, pedagógicas y didácticas de profesores universitarios. Actualidad Educativa, 2(7), pp. 23-29

GENÉ, A. (1991). Cambio conceptual y metodológico en la enseñanza y el aprendizaje de la evolución de los seres vivos. Un ejemplo concreto. Enseñanza de las Ciencias, 9(1), pp. 26-33.

GIL PÉREZ, D. (1983). Tres paradigmas básicos en la enseñanza de las ciencias. Enseñanza de las Ciencias, 1(1), pp. 26-33.

GIL PÉREZ, D. y CARRASCOSA ALLIS, J. (1985). Science learning as conceptual and methodological change. European Journal Science Education, 7(3).

INHELDER, B. y PIAGET, J. (1972). De la lógica del niño a la lógica del adolescente. Buenos Aires: Paidós.

KUHN, T. S. (1972). Laestructura de las revoluciones científicas. México: Fondo de Cultura Económica.

LAKATOS, I. (1983). La metodología de los programas de investigación científica. Madrid: Alianza Editorial.

LANGDE SILVEIRA, E. y MOREIRA, M. A. (1996). Validación de un test para verificar si el alumno posee concepciones científicas sobre calor temperatura y energía interna. Enseñanza de las Ciencias, 14(1), pp. 78-85.

LEDERMAN, N.G. (1992). Students and teachers conceptions of the nature of science: A review of the research. Journal of Research in Science Teaching, 29, pp. 331-359.

MARTÍNEZ OLIVO, J. M. (1996). Estudio sobre la consistencia de las ideas de los alumnos en ciencia. Enseñanza de las Ciencias, 14(1), pp. 87-91.
MELLADO JIMÉNEZ, V. (1996). Concepciones y prácticas del aula de profesores de ciencia, en formación inicial de primaria y secundaria. Enseñanza de las Ciencias, 14(3), pp. 289-302.

NOVAK, J.D. (1981). Applying learning psychology and philosophy of science to biology teaching. The American Biology Teacher, 43(I).

NOVAK, J. D. y GOWIN, D. (1988). Aprendiendo a aprender. Barcelona: Martínez Roca Editores.

PÉREZ GÓMEZ, A.I. (1987). El pensamiento del profesor, vínculo entre la teoría y la práctica. Revista de Educación, 284, pp. 199-221.

PIAGET, J. (1985). Seis estudios de psicología. Barcelona: Planeta-Agostini.

POPPER, K. (1962). La lógica de la investigación científica Madrid: Ediciones Tecnos.

PORLAN ARIZA, R. (1989). «Teoría del conocimiento, teoría de la enseñanza y desarrollo profesional. Las concepciones epistemológicas de los profesores». Tesis doctoral. Sevilla, Universidad de Sevilla. Departamento de Didáctica de las Ciencias.

PORLAN ARIZA, R., RIVERO GARCÍA, R. y MARTÍN DEL POZO, R. 1997. Conocimiento profesional y epistemología de los profesores I: Teoría e instrumentos. Enseñanza de las Ciencias, 15(2), pp. 155-171.

POSNER, G.J., STRIKE, K.A., HEWSON, P.W. y GERTZOG, W.A. (1982). Accommodation of a Scientific conception: Toward a theory conceptual change. Science Education, 66(2), pp. 211-227.

SKINNER, B.F. (1986). Sobre el conductismo. Barcelona: Orbis.

THUILLER, P. (1975). La manipulación de la ciencia. Madrid: Fundamentos.

VIGOTSKI,L.S.(1989).El desarrollo de los procesospsicológicos superiores. Barcelona: Crítica.

[Artículo recibido en septiembre de 2000 y aceptado en abril de 2002.] 


\section{ANEXO I}

Tabla I

Características del grupo con el cual se hizo la investigación.

\begin{tabular}{|c|c|c|c|c|}
\hline Estudiante & Género & Edad (años) & Título & Experiencia docente \\
\hline 01 & Femenino & 29 & Lic. en Química. 1992 & 11 años. Química. Sec. \\
\hline 02 & Femenino & 36 & Lic. en Química y biología. 1986 & 6 años. Química y biología \\
\hline 03 & Femenino & 33 & Lic. en Biología y química. 1992 & 4 años. Química y biología. Sec. \\
\hline 04 & Masculino & & Lic. en Química. 1975 & 20 años. Química. Sec. \\
\hline 05 & Femenino & 30 & Lic. en Química. 1992 & 5 años. Biología y química. Sec. \\
\hline 06 & Masculino & 48 & Lic. en Química. 1977 & 24 años. Química \\
\hline 07 & Femenino & 40 & Lic. en Química. 1977 & 19 años. Biología y química. Sec. \\
\hline 08 & Masculino & 42 & Lic. en Química. 1976 & 20 años. Química y estadística. Sec. \\
\hline 09 & Femenino & 42 & Lic. en Química. 1982 & 20 años. Química y biología. Sec. \\
\hline 10 & Masculino & 32 & Lic. en Biología. 1990 & 2 años. Biología y química. Sec. \\
\hline 11 & Femenino & 24 & Lic. en Química. 1995 Química. Universidad \\
\hline 12 & Femenino & 28 & Lic. en Química. 1995 & 11 años. Básica primaria \\
\hline 13 & Femenino & 33 & Lic. en Química. 1991 & 6 años. Química y biología. Sec. \\
\hline 14 & Femenino & 28 & Lic. en Química & 17 años. Biología y química. Sec. \\
\hline 15 & Masculino & 40 & Lic. en Química.1981 & 16 años. Química y matemáticas. \\
\hline 16 & Masculino & 24 & Lic. en Química. 1995 & 3 años. Química. Sec. \\
\hline 17 & Masculino & 24 & Lic. en Química. 1995 & 2 años. Química y biología. Sec. \\
\hline
\end{tabular}




\section{ANEXO II}

Prueba tipo Likert general.

\section{A. INSTRUCCIONES}

A continuación usted encontrará una serie de afirmaciones numeradas de 1 a 50 sobre las cuales se solicita su posición de conformidad con la escala:

1) Totalmente de acuerdo. 2) De acuerdo. 3) No sé qué decir. 4) En desacuerdo. 5) Totalmente en desacuerdo.

Marque en las casillas correspondientes a cada afirmación, en la hoja de respuestas, la que corresponda a su parecer según el orden de esta escala. Por favor, no dañe este cuadernillo.

Los resultados serán utilizados con propósitos eminentemente investigativos, por lo que los nombres en las hojas de respuestas tendrán una función de control y no serán mencionados bajo ninguna consideración.

\section{B. AFIRMACIONES}

1. Enseñar es transmitir conocimientos a los estudiantes.

2. Las teorías de las ciencias se obtienen a partir de observaciones cuidadosas del mundo.

3. Los estudiantes demuestran su aprendizaje cuando repiten lo expuesto en clase.

4. La tarea de los hombres de ciencias es construir hipótesis y sistemas de hipótesis sobre la realidad.

5.Todo aprendizaje lo es en la medida en que los alumnos elaboran nuevos significados.

6. Para enseñar, el profesor hace que los estudiantes observen algunos fenómenos y saquen conclusiones.

7. Sobre los contenidos a enseñar los alumnos carecen de ideas al respecto.

8. Los experimentos son construcciones instrumentales que preceden a las teorías.

9. Lo más importante para el profesor es hacer explicaciones en clase.

10. Las teorías de las ciencias son tramados significativos de conceptos.

11. Los estudiantes aprenden a partir de lo que ya saben.

12. Uno de los aspectos de la enseñanza es procurar que los estudiantes contrasten, mediante observaciones, los significados que han elaborado.

13. Los profesores realizan su tarea de acuerdo con sus concepciones epistemológicas, pedagógicas y didácticas.

14. Las teorías científicas son fruto de procesos inductivos.

15. Aprender es transformar aquellas explicaciones que ya se poseen sobre los fenómenos acerca de los cuales trata el contenido de la clase.

16. Lo que caracteriza a la ciencia no son los hechos sino sus teorías sobre los mismos.

17. Para enseñar no es indispensable lo que los alumnos ya saben.

18. Para ser profesor es suficiente el dominio de los contenidos de la asignatura.

19. El progreso científico se caracteriza por la continuidad y conservación de las ideas sobre el mundo.

20. El aprendizaje es siempre una acumulación de información.

21. Al enseñar, el profesor debe perseguir un cambio conceptual, metodológico, actitudinal y axiológico.

22. La pedagogía y la didáctica de un saber se traduce en recomendaciones de cómo realizar una buena clase.

23. Enseñar es organizar el ámbito pedagógico y didáctico con miras a lograr unas experiencias de aprendizaje.

24. La historia de las ciencias da cuenta de cómo, en diferentes épocas, los científicos han sostenido y cambiado sus diferentes teorías y realizaciones.

25. Aprender es aceptar una serie de verdades absolutas.

26. Cuando enseñan, los profesores tienen en cuenta sus concepciones sobre el aprendizaje.

27. Aprender significativamente no implica cambiar lo que ya se sabe.

28. Enseñar es procurar que el alumno asimile lo expuesto por el profesor.

29. Los conceptos de la ciencias son construcciones que luego se contrastan con la realidad. 
30. Enseñar es lograr que los alumnos descubran el conocimiento.

31. Concepciones pedagógicas y didácticas rigurosas cambian los procesos de enseñanza y de aprendizaje.

32. El conocimiento científico es una permanente acumulación de principios, leyes y teorías.

33. En el proceso de aprendizaje, los estudiantes construyen, conscientemente, nuevos significados.

34. El mejor profesor es aquél que logra que sus estudiantes adquieran más conocimientos.

35. La tarea de la investigación científica es descubrir cómo verdaderamente funciona el mundo.

36. El estudiante demuestra que ha aprendido cuando reproduce lo que el profesor le ha enseñado.

37. Enseñar es desarrollar en los estudiantes la conciencia de que los conceptos científicos cambian.

38. Las ciencias son una tradición cambiante de lenguajes y metodologías.

39. La preocupación de los profesores es enseñar a leer y escribir a los estudiantes en sus disciplinas.

40. El aprendizaje es un proceso constructivo en el cual el estudiante se aproxima, en forma paulatina, a las conceptualizaciones y las metodologías de una comunidad de especialistas.

41. Los hombres y las mujeres de ciencias se dedican a la construcción de teorías sobre el mundo.

42. En la enseñanza de las ciencias, los experimentos buscan demostrar los logros teóricos elaborados por los estudiantes.

43. El aprendizaje es independiente en la enseñanza.

44. Los conceptos científicos se encuentran en las cosas y fenómenos de la realidad.

45. El mejor profesor es aquél que logra un cambio conceptual, metodológico, actitudinal y axiológico en sus estudiantes.

46. Los estudiantes poseen concepciones sobre aquello que el profesor pretende enseñarles.

47. A las teorías se llega por descubrimiento.

48. Al enseñar, el profesor busca que sus estudiantes construyan teorías que luego deben demostrar

49. El estudiante aprende a pensar y aun en contra de la enseñanza del profesor.

50. Los hombres y mujeres de ciencia se dedican a repetir lo validado por la tradición. 


\section{ANEXO III}

Prueba tipo Likert de química.

\section{A. INSTRUCCIONES}

A continuación encontrará usted una serie de afirmaciones numeradas de 1 a 50 sobre las cuales se solicita su posición de conformidad con la escala:

1) Totalmente de acuerdo. 2) De acuerdo. 3) No sé qué decir. 4) En desacuerdo. 5) Totalmente en desacuerdo.

Marque con una X en las casillas correspondientes a cada afirmación, en la hoja de respuestas, la que corresponda a su parecer según el orden de la escala. Por favor, no escriba nada en este cuadernillo.

Los resultados serán utilizados con propósitos eminentemente investigativos, por lo que su nombre en la hoja de respuestas tendrá una función de control y no será mencionado bajo ninguna consideración.

\section{B. AFIRMACIONES}

1. Los químicos se dedican a elaborar teorías sobre la composición, la estructura y las propiedades de las sustancias.

2. El conocimiento químico se encuentra en la naturaleza.

3. La química es un caso particular de la física.

4. La principal guía en la producción de saber químico es el método científico.

5. A partir de la observación empírica se inducen las leyes y principios de la química.

6. Mediante el experimento químico se corroboran las hipótesis en esta ciencia.

7. Los conceptos químicos son matemáticos e instrumentales a la vez.

8. La química trata de las interacciones sustancia-sustancia, sustancia-energía.

9. Las propiedades químicas se manifiestan en las reacciones.

10. Entre los químicos existen varios métodos científicos.

11. La teoría química propiamente dicha es la del enlace.

12. El conocimiento químico es una construcción histórica de los especialistas en esta ciencia.

13. La producción de saber en química es fruto de programas de investigación.

14. Los experimentos químicos se realizan aun en ausencia de una teoría.

15. El proceso de aprendizaje de la química es una memorización y repetición de las teorías y métodos vigentes.

16. Las propiedades físicas de las sustancias miden las interacciones de éstas con las distintas formas de energía.

17. Históricamente la química es una acumulación de descubrimientos.

18. Para hacer aparecer la materia en términos de sustancias y de mezclas, le basta al químico con observar la naturaleza.

19. Una enseñanza significativa de esta ciencia persigue cambiar lo que los alumnos ya saben al respecto.

20. Diferentes grupos de químicos poseen ideas diferentes sobre el mismo tema que trabajan.

21. La herramienta fundamental de los químicos es la dialéctica del análisis-síntesis.

22. La lógica que siguen los miembros de esta comunidad de especialistas es la deductiva (de lo general a lo particular).

23. Los experimentos químicos y los experimentos en física son semejantes.

24. Cuando se va a enseñar química, aquello que los alumnos ya saben carece de importancia.

25. El aprendizaje de esta ciencia es un proceso acumulativo de nuevas ideas.

26. La precisión de los datos que se obtienen en un experimento químico está determinada por los instrumentos que se utilizan.

27. En toda reacción química, reactantes y productos están separados.

28. El peso molecular es el peso en gramos de una mol de moléculas.

29. Los químicos se dedican a descubrir las sustancias en la naturaleza. 
30. Toda observación hecha por los químicos está precedida de una sede.

31. Las sustancias que hacen parte del aval químico han sido primero formuladas teóricamente.

32. En los experimentos químicos se contrastan instrumentalmente las predicciones sobre las interacciones sustancia-sustancia y sustancia-energía.

33. Los estudiantes aprenden la química construyendo nuevos significados.

34. La enseñanza de la química se centra en la transmisión de conocimientos.

35. Los conceptos químicos son una construcción histórica de esta comunidad de especialistas.

36. Los químicos deducen desde sus teorías las interacciones sustancia-sustancia, sustancia-energía a determinar en los experimentos.

37. El problema central de las comunidades de químicos es la molecularidad.

38. La acidez de una sustancia depende de otra sustancia de frente a la cual se encuentre.

39. Para enseñar química, el profesor ha de partir de la observación de sus estudiantes.

40. Cuando se aprende significativamente la química, se hace sin intervención de lo que ya se sabe.

41. Para sintetizar una sustancia es indispensable diseñarla conceptual y metodológicamente primero en el papel.

42. Para corroborar que una estructura propuesta es la que puede hacer aparecer una sustancia, hay que combinar sus análisis y su síntesis.

43. La química teórica y la química aplicada conforman una unidad conceptual, metodológica, actitudinal y axiológica.

44. La conversión de la categoría filosófica de átomo en un concepto científico se debió a la racionalidad química.

45. El conocimiento químico ha permanecido inmodificable desde Lavoisier.

46. Todas las comunidades químicas se orientan por las mismas concepciones conceptuales, metodológicas y actitudinales.

47. La existencia de verdaderas teorías químicas es discutible.

48. La enseñanza es propiciar la construcción de significados nuevos en los estudiantes.

49. Los distintos programas de investigación en química siguen las mismas orientaciones conceptuales y metodológicas.

50. El aprendizaje de la química es la repetición al pie de la letra de lo enseñado por el profesor. 


\section{ANEXO IV}

\section{PRUEBA SEMÁNTICA}

Nombre: Fecha:

A continuación se le suministra una serie de términos. Anote en cada caso, lo que para usted significa cada uno de ellos.

Ciencia, saber, conocimiento, experimento, método, prueba, enseñanza, aprendizaje, información, construcción, empiria, verificar, contrastar, epistemología, corroboración, reglas de producción, objeto de conocimiento, descubrimiento, teoría y química.

\section{ANEXO V}

\section{CUESTIONARIO}

Por favor, responda usted las siguientes preguntas:

1. ¿Cómo ha sido su desempeño docente?

2. Después de un año de posgrado, ¿cuál cree usted ha sido su cambio más importante?

3. ¿Qué conceptos tienen usted de las pruebas tipo Likert, de la de significados, la de composición y la de los mapas conceptuales que se le aplicaron y elaboró?

4. Indique cómo procedería con sus estudiantes frente a una temática de química a enseñar y cómo contrastaría el aprendizaje logrado por ellos. 